\title{
Sampled-Data Observer-Based Decentralized Fuzzy Control for Nonlinear Large-Scale Systems
}

\author{
Geun Bum Koo*, Jin Bae Park ${ }^{\dagger}$ and Young Hoon Joo**
}

\begin{abstract}
In this paper, a sampled-data observer-based decentralized fuzzy control technique is proposed for a class of nonlinear large-scale systems, which can be represented to a Takagi-Sugeno fuzzy system. The premise variable is assumed to be measurable for the design of the observer-based fuzzy controller, and the closed-loop system is obtained. Based on an exact discretized model of the closed-loop system, the stability condition is derived for the closed-loop system. Also, the stability condition is converted into the linear matrix inequality (LMI) format. Finally, an example is provided to verify the effectiveness of the proposed techniques.
\end{abstract}

Keywords: Sampled-data observer-based decentralized fuzzy control, Nonlinear large-scale systems, Takagi-Sugeno fuzzy system, Exact discretized model, Linear matrix inequality

\section{Introduction}

Recently, as network-based systems are more increased in many engineering applications, such as wireless sensor networks, smart spaces and wide-area power systems, large-scale sampled-data systems, which well-represent characteristics of a network-based system, have attracted much attention $[1,2]$. First, in the case of the large-scale system, due to the interconnection among subsystems, various problems are encountered, such as the high dimensionality and the structure constraint of the controller. To control a large-scale system, the decentralized control technique is more suitable than the traditional centralized control one. Thus, many decentralized control techniques have been proposed [3-7]; the decentralized fuzzy control technique [12-15] using a Takagi-Sugeno (T-S) fuzzy model has been recognized as one of the predominant decentralized control methods.

Apart from the large-scale system issue, the sampleddata control system has both a continuous-time plant and a discrete-time controller. In the case of linear systems, because the exact discretization of the continuous-time plant is possible, the sampled-data controller design is not difficult. However, in the case of nonlinear systems, the exact discretization is not possible, and so, to overcome this problem, various nonlinear sampled-data control techniques have been proposed, such as the Euler approximation [8, 9] and the conversion to an input delay controller [10, 11]. Sampled-data fuzzy control techniques, which are combination of the sampled-data control technique and the

$\dagger$ Corresponding Author: Dept. of Electrical and Electronic Engineering, Yonsei University, Korea. (jbpark@yonsei.ac.kr)

* Dept. of Electrical and Electronic Engineering, Yonsei University, Korea. (milbam@yonsei.ac.kr)

** Dept. of Control and Robotics Engineering, Kunsan National University, Korea. (yhjoo@kunsan.ac.kr)

Received: November 18, 2014; Accepted: November 3, 2015
T-S fuzzy model, have also been proposed in many studies [16-27] and are categorized into the input delay conversion approach and the direct discrete-time design approach.

First, the input delay conversion approach is to convert a sampled-data fuzzy controller into a continuous-time fuzzy one with the input time delay. This approach has been used in many studies [16-19], with such methods as $H_{\infty}$ control [18] and robust control [19]. However, there are few studies about observer-based control or decentralized control in the input delay conversion approach. The direct discrete-time design approach [20-27] is to guarantee stability using the discretized model of the continuous-time plant. In [20-22], various sampled-data fuzzy control techniques using the direct discrete-time design approach have been proposed, such as the observer-based outputfeedback scheme [20] and the robust control [21]. However, these studies did not address the problem of discretized errors. To conquer this problem, the analysis of the stability of the discrete-time model considering the discretized error and the limitation analysis of stability for the approximately discretized model have been studied in [23, 24]. In [25], by using the exact discretized model, a sampled-data fuzzy controller was designed for stabilization of the nonlinear system. The methodology of [25] was extended to the observer-based output-feedback scheme [26] and the guaranteed cost control technique [27], but the sampleddata decentralized fuzzy control problem has not been studied yet. Thus, there still remain sampled-data fuzzy control issues for the decentralized and the observer-based output-feedback approaches.

Motivated by the aforementioned analysis, this paper presents the sampled-data observer-based decentralized fuzzy control technique for a nonlinear large-scale system. Using the T-S fuzzy model, the nonlinear large-scale system is represented as a fuzzy large-scale system, and the observer-based controller is assumed to have the measurable 
premise variable. Based on the exact discretized model and the discrete-time Lyapunov functional, the sufficient condition of the stabilization is investigated for closed-loop system. Also, the stability condition is converted into linear matrix inequality (LMI) formats [32-36]. Finally, by the example, the validity of the proposed ideas, techniques, and procedures is shown.

Notation: The subscripts $i$ and $j$ denote the fuzzy rule indices and the subscripts $k$ and $l$ denote the subsystem indices. The notation $(\cdot)^{T}$ and $*$ are used for the transpose of the argument and the transposed element in symmetric positions, respectively. The notation $\sum_{l \neq k}^{q}$ means $\sum_{l=1, l \neq k}^{q}$.

\section{Preliminaries}

Consider a T-S fuzzy large-scale system consisting of $q$ subsystems. Then, the $i$ th IF-THEN rule of the $k$ th subsystem is represented by the following form:

$$
\begin{gathered}
R_{i}^{k}: I F z_{k_{1}} \text { is } \Gamma_{i 1}^{k} \text { and } \ldots \text { and } z_{k_{p}} \text { is } \Gamma_{i p}^{k}, \\
\text { THEN }\left\{\begin{array}{l}
\dot{x}_{k}(t)=A_{i}^{k} x_{k}(t)+B_{i}^{k} u_{k}(t)+\sum_{l \neq k}^{q} A_{i}^{k l} x_{l}(t) \\
y_{k}(t)=y_{k}(n T)=C_{i}^{k} x_{k}(n T)
\end{array}\right.
\end{gathered}
$$

where $z_{k_{m}}, m \in \mathcal{I}_{p}$ is the premise variable; $x_{k} \in \mathbb{R}^{n_{k}}$ is the state variable; $u_{k} \in \mathbb{R}^{n_{k}}$ is the control input variable; $y_{k} \in \mathbb{R}^{\beta_{k}}$ is the sampled-data output variable to be determined in the time interval $t \in[n T, n T+T), k \in \mathbb{Z}_{\underline{1} 0}$; $\Gamma_{i m}^{k},(i, m, k) \in \mathcal{I}_{r} \times \mathcal{I}_{p} \times \mathcal{I}_{q}$, is a fuzzy set for $z_{k_{m}} ; A_{i}^{k}$, $B_{i}^{k}$ and $C_{i}^{k}$ denote nominal system matrices with appropriate dimensions for the $i$ th rule of the $k$ th subsystem; and $A_{i}^{k l}$ is the interconnection matrix between the $k$ th and the $l$ th subsystems.

By applying the center-average defuzzification, product inference, and singleton fuzzifier into the fuzzy IF-THEN rule (1), the $k$ th subsystem of the nonlinear large-scale system can be inferred as follows:

$$
\begin{aligned}
& \dot{x}_{k}(t)=\sum_{i=1}^{r} \mu_{i}^{k}\left(z_{k}(t)\right)\left(A_{i}^{k} x_{k}(t)+B_{i}^{k} u_{k}(t)+\sum_{l \neq k}^{q} A_{i}^{k l} x_{l}(t)\right) \\
& y_{k}(t)=y_{k}(n T)=\sum_{i=1}^{r} \mu_{i}^{k}\left(z_{k}(n T)\right) C_{i}^{k} x_{k}(n T)
\end{aligned}
$$

where

$$
\begin{gathered}
\mu_{i}^{k}\left(z_{k}(t)\right)=\omega_{i}^{k}\left(z_{k}(t)\right) / \sum_{i=1}^{r} \omega_{i}^{k}\left(z_{k}(t)\right), \\
\omega_{i}^{k}\left(z_{k}(t)\right)=\prod_{m=1}^{p} \Gamma_{i m}^{k}\left(z_{m}(t)\right)
\end{gathered}
$$

in which $\Gamma_{i m}^{k}\left(z_{k_{m}}(t)\right)$ is the fuzzy membership grade of $z_{k_{m}}$ in $\Gamma_{i m}^{k}$.
Assumption 1: The state variable $x_{k}(t)$ is not measurable, but the premise variable $z_{k}(t)$ is measurable in the continuous-time sense and the output variable $y_{k}(t)$ is measurable only at sampling instants.

Depending on Assumption 1, we consider the following sampled-data observer-based decentralized fuzzy controller:

$$
\begin{aligned}
\dot{\hat{x}}_{k}(t) & =\sum_{i=1}^{r} \mu_{i}^{k}\left(z_{k}(t)\right)\left(A_{i}^{k} \hat{x}_{k}(t)+B_{i}^{k} u_{k}(t)+L_{i}^{k}\left(y_{k}(t)-\hat{y}_{k}(t)\right)\right) \\
& =: A^{k}(t) \hat{x}_{k}(t)+B^{k}(t) u_{k}(t)+L^{k}(t)\left(y_{k}(t)-\hat{y}_{k}(t)\right) \\
\hat{y}_{k}(t) & =\hat{y}_{k}(n T)=\sum_{i=1}^{r} \mu_{i}^{k}\left(z_{k}(n T)\right) C_{i}^{k} \hat{x}_{k}(n T)=: C^{k}(n T) \hat{x}_{k}(n T) \\
u_{k}(t) & =u_{k}(n T)=\sum_{i=1}^{r} \mu_{i}^{k}\left(z_{k}(n T)\right) K_{i}^{k} \hat{x}_{k}(n T)=: K^{k}(n T) \hat{x}_{k}(n T)
\end{aligned}
$$

where $\hat{x}_{k}(t)$ is the state variable by the fuzzy observer, respectively; $\hat{y}_{k}(t)$ is the observer output; and $K_{i}^{k}$ and $L_{i}^{k}$ denote the control and observer gains.

To represent the closed-loop system with the sampleddata observer-based decentralized fuzzy controller, we suppose the estimation error $e_{k}(t)=: x_{k}(t)-\hat{x}_{k}(t)$. Then, substituting (3) into (2) and the time derivative of $e_{k}(t)$, the $k$ th sub-closed-loop system can be established as follows:

$$
\dot{\chi}_{k}(t)=\Phi^{k}(t) \chi_{k}(n T)+\Lambda^{k}(t) \tilde{\chi}_{k}(t)+\sum_{l \neq k}^{q} \tilde{A}^{k l}(t) x_{l}(t)
$$

where

$$
\begin{aligned}
\chi_{k}(t) & =\left[\begin{array}{l}
x_{k}(t) \\
e_{k}(t)
\end{array}\right], \quad \tilde{\chi}_{k}(t)=\chi_{k}(t)-\chi_{k}(n T), \\
\Phi^{k}(t) & =\left[\begin{array}{cc}
A^{k}(t)+B^{k}(t) K^{k}(n T) & -B^{k}(t) K^{k}(n T) \\
0 & A^{k}(t)+L^{k}(t) C^{k}(n T)
\end{array}\right], \\
\Lambda^{k}(t) & =\left[\begin{array}{cc}
A^{k}(t) & 0 \\
0 & A^{k}(t)
\end{array}\right], \quad \tilde{A}^{k l}(t)=\left[\begin{array}{l}
A^{k l}(t) \\
A^{k l}(t)
\end{array}\right] .
\end{aligned}
$$

From the $k$ th sub-closed-loop system (4), the observerbased decentralized fuzzy control problem can be stated as follows:

Problem 1: Find the fuzzy observer and control gain matrices $L_{i}^{k}$ and $K_{i}^{k}$ stabilizing the whole closed-loop large-scale system, which are respectively composed of sub-closed-loop systems with the sampled-data observerbased decentralized fuzzy controller.

\section{Main Results}

Before proceeding to the main results, the following lemmas and propositions will be needed throughout the 
proof:

Lemma 1 [28]: Given any function vector $x$, matrix $P=P^{T} \succ 0$, and $t_{0}, t_{f} \in \mathbb{R}$ with $t_{0}<t_{f}$, we have

$$
\left(\int_{t_{0}}^{t_{f}} x(\tau) d \tau\right)^{T} P\left(\int_{t_{0}}^{t_{f}} x(\tau) d \tau\right) \leq\left(t_{f}-t_{0}\right) \int_{t_{0}}^{t_{f}} x(\tau)^{T} P x(\tau) d \tau
$$

Lemma 2 [27]: Suppose the nonlinear system such as $\dot{x}=f(t, x)$, where $f:[n T, n T+T) \times \mathbb{R}^{n}$ is piecewise continuous in $t$ and locally Lipschitz in $x$ and the matrix $P=P^{T} \succ 0$, then the following inequality is always satisfied

$$
\int_{n T}^{n T+T}(x(t)-x(n T))^{T} P(x(t)-x(n T)) d t \leq T^{2} \int_{n T}^{n T+T} \dot{x}(t)^{T} P \dot{x}(t) d t .
$$

Lemma 3 [29]: Given any matrices $Y$ and $P=P^{T} \succ 0$, we have

$$
-Y^{T} P^{-1} Y \leq P-Y^{T}-Y
$$

Proposition 1: In the closed-loop system (4), there exists some constant $\rho>0$ such that

$$
\sum_{k=1}^{q}\left\|x_{k}(t)\right\| \leq \rho \sum_{k=1}^{q}\left\|\chi_{k}(n T)\right\|
$$

for $t \in[n T, n T+T)$.

Proof: From the closed-loop system (4), we have

$$
\begin{gathered}
\dot{x}_{k}(t)=A^{k}(t) x_{k}(t)+B^{k}(t) K^{k}(n T) x_{k}(n T) \\
-B^{k}(t) K^{k}(n T) e_{k}(n T)+\sum_{l \neq k}^{q} A^{k l}(t) x_{l}(t)
\end{gathered}
$$

Integrating from $n T$ to $t$, taking the norm and summing all subsystems on both sides of equation (6) yields

$$
\begin{aligned}
& \sum_{k=1}^{q}\left\|x_{k}(t)\right\| \\
\leq & \sum_{k=1}^{q}\left\|x_{k}(n T)\right\|+\sum_{k=1}^{q} \| \int_{n T}^{t}\left(A^{k}(\tau) x_{k}(\tau)+B^{k}(\tau) K^{k}(n T) x_{k}(n T)\right. \\
& \left.\quad B^{k}(\tau) K^{k}(n T) e_{k}(n T)+\sum_{l \neq k}^{q} A^{k l}(\tau) x_{l}(\tau)\right) d \tau \| \\
\leq & \sum_{k=1}^{q}\left\|x_{k}(n T)\right\|+\sum_{k=1}^{q} \int_{n T}^{t} a\left\|x_{k}(\tau)\right\|+b\left\|x_{k}(n T)\right\| \\
& +b\left\|e_{k}(n T)\right\|+c \sum_{l \neq k}^{q}\left\|x_{l}(\tau)\right\| d \tau
\end{aligned}
$$

where

$$
a=\sup _{(k, i) \mathbb{I}_{q} \times I_{r}}\left\|A_{i}^{k}\right\|, \quad b=\sup _{(k, i, j) \in I_{q} \times I_{r} \times I_{r}}\left\|B_{i}^{k} K_{j}^{k}\right\|,
$$

$$
c=\sup _{(k, l, i) \in I_{q} \times I_{w} \times I_{r}}\left\|A_{i}^{k l}\right\|
$$

and $\mathcal{I}_{q} \times \mathcal{I}_{w}$ denotes all pairs $(k, i) \in \mathcal{I}_{q} \times \mathcal{I}_{q}$ such that $k \neq l$.

By $\sum_{k=1}^{q} \sum_{l \neq k}^{q} x_{l}(t)=(q-1) \sum_{k=1}^{q} x_{k}(t)$, inequality (7) can be further developed as follows:

$$
\begin{aligned}
\sum_{k=1}^{q}\left\|x_{k}(t)\right\| & \leq \sum_{k=1}^{q}\left((1+T b)\left\|x_{k}(n T)\right\|+T b\left\|e_{k}(n T)\right\|\right) \\
& +(a+(q-1) c) \int_{n T}^{t} \sum_{k=1}^{q}\left\|x_{k}(\tau)\right\| d \tau \\
& \leq \sqrt{(1+T b)^{2}+(T b)^{2}} \sum_{k=1}^{q}\left\|\chi_{k}(n T)\right\| \\
& +(a+(q-1) c) \int_{n T}^{t} \sum_{k=1}^{q}\left\|x_{k}(\tau)\right\| d \tau
\end{aligned}
$$

Then, an application of the Gronwall-Bellman inequality to $\sum_{k=1}^{q}\left\|x_{k}(t)\right\|$ results in

$$
\begin{aligned}
& \sum_{k=1}^{q}\left\|x_{k}(t)\right\| \\
& \leq \sqrt{(1+T b)^{2}+(T b)^{2}} \exp ((a+(q-1) c) T) \sum_{k=1}^{q}\left\|\chi_{k}(n T)\right\| . \\
& =\rho \sum_{k=1}^{q}\left\|\chi_{k}(n T)\right\| .
\end{aligned}
$$

Remark 1: Proposition 1 shows the relation between $\sum_{k=1}^{q}\left\|x_{k}(t)\right\|$ and $\sum_{k=1}^{q}\left\|x_{k}(n T)\right\|$ for the closed-loop system (4). By Proposition 1, we know that each state variable $x_{k}(t)$ converges to the origin when the whole $x_{k}(n T)$ converges to the origin.

The sufficient condition for stability of the closed-loop system (4) is summarized as the following proposition:

Proposition 2: If there exist some symmetric and positive definite matrices $P_{1}^{k}, P_{2}^{k}, P_{3}^{k}$ and some matrices $K_{i}^{k}, L_{i}^{k}$ such that the following inequality is satisfied, then the whole closed-loop system which is composed of sub-closed-loop systems (4) is asymptotically stable.

$$
\left.\begin{array}{ccccc}
-T^{-1} \omega P_{1}^{k}+\tilde{P}_{3}^{k} & * & * & * & * \\
\tilde{P}_{3}^{k} & -P_{2}^{k}+\tilde{P}_{3}^{k} & * & * & * \\
0 & 0 & -P_{3}^{k} & * & * \\
\omega \Psi^{k}(t) & \omega \Lambda^{k}(t) & \tilde{A}^{k}(t)-T^{-1} \omega\left(P_{1}^{k}\right)^{-1} & * \\
\omega \Psi^{k}(t) & \omega \Lambda^{k}(t) & \tilde{A}^{k l}(t) & 0 & -T^{-2} \omega^{2}\left(P_{2}^{k}\right)^{-1}
\end{array}\right] \prec 0
$$

where, $\tilde{P}_{3}^{k}=\operatorname{diag}\left\{P_{3}^{k}, 0\right\}, \Psi^{k}(t)=T^{-1} I+\Phi^{k}(t), \omega=(q-1)^{-1}$ 
for $t \in[n T, n T+T)$.

Proof: By integrating the closed-loop system (4) from $n T$ to $n T+T$, we have the exact discretized model as follows:

$$
\begin{aligned}
& \chi_{k}(n T+T) \\
& =\chi_{k}(n T)+\int_{n T}^{n T+T}\left(\Phi^{k}(t) \chi_{k}(n T)+\Lambda^{k}(t) \tilde{\chi}_{k}(t)+\sum_{l \neq k}^{q} \tilde{A}^{k l}(t) x_{l}(t)\right) d t
\end{aligned}
$$

Based on the discretized model (9), we consider the discrete-time Lyapunov function candidate as follows:

$$
V=\sum_{k=1}^{q} V_{k}\left(\chi_{k}(n T)\right)=\sum_{k=1}^{q} \chi_{k}(n T)^{T} P_{1}^{k} \chi_{k}(n T) .
$$

where $P_{1}^{k}=\left(P_{1}^{k}\right)^{T} \succ 0$, then the first forward difference of $V$ can be defined by

$\Delta V=\sum_{k=1}^{q}\left(\chi_{k}(n T+T)^{T} P_{1}^{k} \chi_{k}(n T+T)-\chi_{k}(n T)^{T} P_{1}^{k} \chi_{k}(n T)\right)$.

Substituting (9) into (10) yields

$$
\begin{aligned}
& \Delta V=\sum_{k=1}^{q}\left(\chi_{k}(n T)+\int_{n T}^{n T+T}\left(\Phi^{k}(t) \chi_{k}(n T)+\Lambda^{k}(t) \tilde{\chi}_{k}(t)\right.\right. \\
& \left.\left.\quad+\sum_{l \neq k}^{q} \tilde{A}^{k l}(t) x_{l}(t)\right) d t\right)^{T} P_{1}^{k} \\
& \quad \times\left(\chi_{k}(n T)+\int_{n T}^{n T+T}\left(\Phi^{k}(t) \chi_{k}(n T)+\Lambda^{k}(t) \tilde{\chi}_{k}(t)\right.\right. \\
& \left.\left.\quad+\sum_{l \neq k}^{q} \tilde{A}^{k l}(t) x_{l}(t)\right) d t\right)-\sum_{k=1}^{q} \chi_{k}(n T)^{T} P_{1}^{k} \chi_{k}(n T) \\
& =\sum_{k=1}^{q}\left(\int_{n T}^{n T+T}\left(\Psi^{k}(t) \chi_{k}(n T)+\Lambda^{k}(t) \tilde{\chi}_{k}(t)+\sum_{l \neq k}^{q} \tilde{A}^{k l}(t) x_{l}(t)\right) d t\right)^{T} \\
& \quad \times P_{1}^{k}\left(\int_{n T}^{n T+T}\left(\Psi^{k}(t) \chi_{k}(n T)+\Lambda^{k}(t) \tilde{\chi}_{k}(t)+\sum_{l \neq k}^{q} \tilde{A}^{k l}(t) x_{l}(t)\right) d t\right) \\
& \quad-\sum_{k=1}^{q} \chi_{k}(n T)^{T} P_{1}^{k} \chi_{k}(n T) .
\end{aligned}
$$

By applying Lemma 1 and 2, equation (11) becomes

$$
\begin{aligned}
\Delta V= & \sum_{k=1}^{q} \int_{n T}^{n T+T}\left(\Psi^{k}(t) \chi_{k}(n T)+\Lambda^{k}(t) \tilde{\chi}_{k}(t)+\sum_{l \neq k}^{q} \tilde{A}^{k l}(t) x_{l}(t)\right)^{T} \\
& \times T P_{1}^{k}\left(\Psi^{k}(t) \chi_{k}(n T)+\Lambda^{k}(t) \tilde{\chi}_{k}(t)+\sum_{l \neq k}^{q} \tilde{A}^{k l}(t) x_{l}(t)\right) d t \\
& -\sum_{k=1}^{q} \chi_{k}(n T)^{T} P_{1}^{k} \chi_{k}(n T)
\end{aligned}
$$

$$
\begin{aligned}
& +\sum_{k=1}^{q} \sum_{l \neq k}^{q} \int_{n T}^{n T+T}\left(\Psi^{k}(t) \chi_{k}(n T)+\Lambda^{k}(t) \tilde{\chi}_{k}(t)+\sum_{l \neq k}^{q} \tilde{A}^{k l}(t) x_{l}(t)\right)^{T} \\
& \times T^{2} P_{2}^{k}\left(\Psi^{k}(t) \chi_{k}(n T)+\Lambda^{k}(t) \tilde{\chi}_{k}(t)+\sum_{l \neq k}^{q} \tilde{A}^{k l}(t) x_{l}(t)\right) d t \\
& -\sum_{k=1}^{q} \sum_{l \neq k}^{q} \int_{n T}^{n T+T} \tilde{\chi}_{k}(t)^{T} P_{2}^{k} \tilde{\chi}_{k}(t) d t
\end{aligned}
$$

where $P_{2}^{k}=\left(P_{2}^{k}\right)^{T} \succ 0$.

For the positive definite matrices $P_{1}^{k}$ and $P_{3}^{k}$, the followings are satisfied

$$
\begin{aligned}
& \sum_{k=1}^{q}\left(\sum_{l \neq k}^{q} \tilde{A}^{k l}(t) x_{l}(t)\right)^{T} P_{1}^{k}\left(\sum_{l \neq k}^{q} \tilde{A}^{k l}(t) x_{l}(t)\right) \\
& \leq(q-1) \sum_{k=1}^{q} \sum_{l \neq k}^{q}\left(\tilde{A}^{k l}(t) x_{l}(t)\right)^{T} P_{1}^{k} \tilde{A}^{k l}(t) x_{l}(t) .
\end{aligned}
$$

$\sum_{k=1}^{q} \sum_{l \neq k}^{q} \int_{n T}^{n T+T} x_{k}(t)^{T} P_{3}^{k} x_{k}(t) d t=\sum_{k=1}^{q} \sum_{l \neq k}^{q} \int_{n T}^{n T+T} x_{l}(t)^{T} P_{3}^{l} x_{l}(t) d t$

By applying (13) and (14) to (12), we have

$$
\begin{aligned}
& \Delta V \leq \sum_{k=1}^{q} \sum_{l \neq k}^{q} \int_{n T}^{n T+T}\left(\omega \Psi^{k}(t) \chi_{k}(n T)+\omega \Lambda^{k}(t) \tilde{\chi}_{k}(t)+\tilde{A}^{k l}(t) x_{l}(t)\right)^{T} \\
& \times T \omega^{-1} P_{1}^{k}\left(\omega \Psi^{k}(t) \chi_{k}(n T)+\omega \Lambda^{k}(t) \tilde{\chi}_{k}(t)+\tilde{A}^{k l}(t) x_{l}(t)\right) d t \\
& -\sum_{k=1}^{q} \sum_{l \neq k}^{q} \int_{n T}^{n T+T} T^{-1} \omega \chi_{k}(n T)^{T} P_{1}^{k} \chi_{k}(n T) d t \\
& +\sum_{k=1}^{q} \sum_{l \neq k}^{q} \int_{n T}^{n T+T}\left(\omega \Psi^{k}(t) \chi_{k}(n T)+\omega \Lambda^{k}(t) \tilde{\chi}_{k}(t)+\tilde{A}^{k l}(t) x_{l}(t)\right)^{T} \\
& \times T^{2} \omega^{-2} P_{2}^{k}\left(\omega \Psi^{k}(t) \chi_{k}(n T)+\omega \Lambda^{k}(t) \tilde{\chi}_{k}(t)+\tilde{A}^{k l}(t) x_{l}(t)\right) d t \\
& -\sum_{k=1}^{q} \sum_{l \neq k}^{q} \int_{n T}^{n T+T} \tilde{\chi}_{k}(t)^{T} P_{2}^{k} \tilde{\chi}_{k}(t) d t \\
& +\sum_{k=1}^{q} \sum_{l \neq k}^{q} \int_{n T}^{n T+T}\left(\chi_{k}(n T)+\tilde{\chi}_{k}(t)\right)^{T} \tilde{P}_{3}^{k}\left(\chi_{k}(n T)+\tilde{\chi}_{k}(t)\right) d t \\
& -\sum_{k=1}^{q} \sum_{l \neq k}^{q} \int_{n T}^{n T+T} x_{l}(t)^{T} P_{3}^{l} x_{l}(t) d t \\
& =\sum_{k=1}^{q} \sum_{l \neq k}^{q} \int_{n T}^{n T+T}\left[\begin{array}{c}
\chi_{k}(n T) \\
\tilde{\chi}_{k}(t) \\
x_{l}(t)
\end{array}\right]^{T} \mathcal{P}^{k l}\left[\begin{array}{c}
\chi_{k}(n T) \\
\tilde{\chi}_{k}(t) \\
x_{l}(t)
\end{array}\right] d t \\
& +\sum_{k=1}^{q} \sum_{l \neq k}^{q} \int_{n T}^{n T+T}\left[\begin{array}{c}
\chi_{k}(n T) \\
\tilde{\chi}_{k}(t) \\
x_{l}(t)
\end{array}\right]^{T}\left[\begin{array}{lll}
\omega \Psi^{k}(t) & \omega \Lambda^{k}(t) & \tilde{A}^{k l}(t)
\end{array}\right]^{T}
\end{aligned}
$$

where 


$$
\mathcal{P}^{k l}=\left[\begin{array}{ccc}
-T^{-1} \omega P_{1}^{k}+\tilde{P}_{3}^{k} & * & * \\
\tilde{P}_{3}^{k} & -P_{2}^{k}+\tilde{P}_{3}^{k} & * \\
0 & 0 & -P_{3}^{l}
\end{array}\right]
$$

Thus, from inequality (15), if the following inequality is satisfied

$$
\begin{aligned}
& \mathcal{P}^{k l}+\left[\begin{array}{lll}
\omega \Psi^{k}(t) & \omega \Lambda^{k}(t) & \tilde{A}^{k l}(t)
\end{array}\right]^{T} \\
& \times\left(T \omega^{-1} P_{1}^{k}+T^{2} \omega^{-2} P_{2}^{k}\right)\left[\begin{array}{lll}
\omega \Psi^{k}(t) & \omega \Lambda^{k}(t) & \tilde{A}^{k l}(t)
\end{array}\right] \prec 0
\end{aligned}
$$

then the $\Delta V$ is less than 0 .

By using the Schur complement in (16), we can obtain inequality (8). Thus, if inequality (8) is satisfied, the equilibrium point $x_{k}(n T)=0$ of all discretized subclosed-loop systems (9) is asymptotically stable. Also, by Proposition 1, the equilibrium point $x_{k}(t)=0$ of all subclosed-loop systems (4) is also asymptotically stable.

By Proposition 2, we have the stabilization condition of the nonlinear large-scale system (2) with the sampled-data observer-based decentralized fuzzy controller. However, it is difficult to directly solve inequality (8) and obtain the gain matrices $K_{i}^{k}$ and $L_{i}^{k}$. Thus, to convert into LMI format, which is easily solved by a convex optimization toolbox, we respectively define the matrices $P_{1}^{k}$ and $P_{2}^{k}$ without the loss of generality as follows:

$$
\begin{gathered}
P_{1}^{k}=\operatorname{diag}\left\{P_{11}^{k}, P_{12}^{k}\right\} \\
P_{2}^{k}=\operatorname{diag}\left\{P_{21}^{k}, \alpha_{k} P_{12}^{k}\right\} .
\end{gathered}
$$

where $\alpha_{k}>0$ is a given constant scalar.

Based on the newly defined matrices $P_{1}^{k}, P_{2}^{k}$ and Proposition 2, we summarize the LMI condition satisfying inequality (8).

Theorem 1: If there exist some symmetric and positive matrices $Q_{1}^{k}, Q_{2}^{k}, Q_{3}^{k}, P_{12}^{k}$, some symmetric matrices $R_{1}^{k}$, $R_{2}^{k}, R_{3}^{k}$, and some matrices $M_{i}^{k}, N_{i}^{k}$, such that the following LMIs are satisfied, then inequality (8) is also satisfied and the whole closed-loop system (4) is asymptotically stable.

$$
\begin{aligned}
& \mathcal{X}_{i j}^{k l} \prec 0 \quad(k, l, i, j) \in \mathcal{I}_{q} \times \mathcal{I}_{w} \times \mathcal{I}_{r} \times \mathcal{I}_{r} \\
& {\left[\begin{array}{cc}
Y_{i j}^{k} & * \\
J_{i}^{k} & J_{2}^{k}
\end{array}\right] \prec 0(k, i, j) \in \mathcal{I}_{q} \times \mathcal{I}_{r} \times \mathcal{I}_{r}}
\end{aligned}
$$

where

$$
\mathcal{X}_{i j}^{k l}=\left[\begin{array}{ccc}
G_{1}^{k l} & * & * \\
X_{i j}^{k l} & G_{2}^{k} & * \\
G_{3}^{k} & 0 & -Q_{3}^{k}
\end{array}\right]
$$

$$
\begin{aligned}
& G_{1}^{k}=\operatorname{diag}\left\{-T^{-1} \omega Q_{1}^{k},-\beta_{k} Q_{1}^{k},-Q_{2}^{k},-R_{1}^{k},-Q_{3}^{l}\right\}, \\
& G_{2}^{k}=\operatorname{diag}\left\{-T^{-1} \omega Q_{1}^{k},-R_{2}^{k},-T^{-2} \omega^{2} Q_{2}^{k},-R_{3}^{k}\right\}, \\
& G_{3}^{k}=\left[\begin{array}{lllll}
Q_{1}^{k} & 0 & Q_{2}^{k} & 0 & 0
\end{array}\right],
\end{aligned}
$$$$
X_{i j}^{k}=\left[\begin{array}{ccccc}
\omega\left(T^{-1} Q_{1}^{k}+\Omega_{i j}^{k}\right) & -\omega B_{i}^{k} M_{j}^{k} & \omega A_{i}^{k} Q_{2}^{k} & 0 & A_{i}^{k l} Q_{3}^{l} \\
0 & 0 & 0 & \omega A_{i}^{k} R_{1}^{k} & A_{i}^{k l} Q_{3}^{l} \\
\omega \Omega_{i j}^{k} & -\omega B_{i}^{k} M_{j}^{k} & \omega A_{i}^{k} Q_{2}^{k} & 0 & A_{i}^{k l} Q_{3}^{l} \\
0 & 0 & 0 & \omega A_{i}^{k} R_{1}^{k} & A_{i}^{k l} Q_{3}^{l}
\end{array}\right],
$$$$
\Omega_{i j}^{k}=A_{i}^{k} Q_{1}^{k}+B_{i}^{k} M_{j}^{k},
$$$$
Y_{i j}^{k}=\left[\begin{array}{cccc}
-T^{-1} \omega P_{12}^{k} & * & * & * \\
0 & -\alpha_{k} P_{12}^{k} & * & * \\
\omega\left(T^{-1} P_{12}^{k}+P_{12}^{k} A_{i}^{k}-N_{i}^{k} C_{j}^{k}\right) & 0 & -T^{-1} \omega P_{12}^{k} & * \\
\omega\left(P_{12}^{k} A_{i}^{k}-N_{i}^{k} C_{j}^{k}\right) & 0 & 0 & -T^{-2} \omega^{2} P_{12}^{k}
\end{array}\right] \text {, }
$$$$
J_{2}^{k}=\operatorname{diag}\left\{I, I, \gamma_{1_{k}} P_{12}^{k}, \gamma_{2_{k}} P_{12}^{k}\right\} \text {, }
$$$$
J_{2}^{k}=\operatorname{diag}\left\{-\beta_{k}^{-1} Q_{1}^{k},-R_{1}^{k}, R_{2}^{k}-2 \gamma_{1_{k}} I, R_{3}^{k}-2 \gamma_{2_{k}} I\right\}
$$

and $\alpha_{k}>0, \beta_{k}>0, \gamma_{k_{1}}>0$ and $\gamma_{k_{2}}>0$ are given constant scalars.

Proof: By substituting (17) and (18) into inequality (8), we obtain

$$
\left[\begin{array}{cc}
H_{11}^{k l} & * \\
H_{21}^{k l}(t) & H_{22}^{k}
\end{array}\right] \prec 0
$$

where

$H_{11}^{k l}=\left[\begin{array}{ccccc}-T^{-1} \omega P_{11}^{k}+P_{3}^{k} & * & * & * & * \\ 0 & -T^{-1} \omega P_{12}^{k} & * & * & * \\ P_{3}^{k} & 0 & -P_{21}^{k}+P_{3}^{k} & * & * \\ 0 & 0 & 0 & -\alpha_{k} P_{12}^{k} & * \\ 0 & 0 & 0 & 0 & -P_{3}^{l}\end{array}\right]$,

$H_{21}^{k l}(t)$

$=\left[\begin{array}{ccccc}\omega\left(T^{-1} I+\Upsilon_{1}^{k}(t)\right) & -\omega B^{k}(t) K^{k}(n T) & \omega A^{k}(t) & 0 & A^{k l}(t) \\ 0 & \omega\left(T^{-1} I+\Upsilon_{2}^{k}(t)\right) & 0 & \omega A^{k}(t) & A^{k l}(t) \\ \omega \Upsilon_{1}^{k}(t) & -\omega B^{k}(t) K^{k}(n T) & \omega A^{k}(t) & 0 & A^{k l}(t) \\ 0 & \omega \Upsilon_{2}^{k}(t) & 0 & \omega A^{k}(t) & A^{k l}(t)\end{array}\right]$,

$H_{22}^{k}=\operatorname{diag}\left\{-T^{-1} \omega\left(P_{11}^{k}\right)^{-1},-T^{-1} \omega\left(P_{12}^{k}\right)^{-1}\right.$,

$\left.-T^{-2} \omega^{2}\left(P_{21}^{k}\right)^{-1},-\alpha_{k} T^{-2} \omega^{2}\left(P_{12}^{k}\right)^{-1}\right\}$,

$\Upsilon_{1}^{k}(t)=A^{k}(t)+B^{k}(t) K^{k}(n T), \quad \Upsilon_{2}^{k}(t)=A^{k}(t)-L^{k}(t) C^{k}(n T)$.

If there exist symmetric matrices $\left(R_{1}^{k}\right)^{-1}, R_{2}^{k}$ and $R_{3}^{k}$ such that the following inequality is satisfied:

$$
\left[\begin{array}{cccc}
-T^{-1} \omega P_{12}^{k} & * & * & * \\
0 & -\alpha_{k} P_{12}^{k} & * & * \\
\omega\left(T^{-1} I+\Upsilon_{2}^{k}(t)\right) & 0 & -T^{-1} \omega\left(P_{12}^{k}\right)^{-1} & * \\
\omega \Upsilon_{2}^{k}(t) & 0 & 0 & -T^{-2} \omega^{2}\left(P_{12}^{k}\right)^{-1}
\end{array}\right]
$$


$\prec\left[\begin{array}{cccc}-\beta_{k} P_{11}^{k} & * & * & * \\ 0 & -\left(R_{1}^{k}\right)^{-1} & * & * \\ 0 & 0 & -R_{2}^{k} & * \\ 0 & 0 & 0 & -R_{3}^{k}\end{array}\right]$

Then, inequality (21) is majorized by

$$
\left[\begin{array}{cc}
\hat{H}_{11}^{k l} & * \\
\hat{H}_{21}^{k l}(t) & \hat{H}_{22}^{k}
\end{array}\right] \prec 0
$$

where

$\hat{H}_{11}^{k l}=\left[\begin{array}{ccccc}-T^{-1} \omega P_{11}^{k}+P_{3}^{k} & * & * & * & * \\ 0 & -\beta_{k} P_{11}^{k} & * & * & * \\ P_{3}^{k} & 0 & -P_{21}^{k}+P_{3}^{k} & * & * \\ 0 & 0 & 0 & -\left(R_{1}^{k}\right)^{-1} & * \\ 0 & 0 & 0 & 0 & -P_{3}^{l}\end{array}\right]$,

$$
\hat{H}_{21}^{k l}(t)
$$$$
=\left[\begin{array}{ccccc}
\omega\left(T^{-1} I+\Upsilon_{1}^{k}(t)\right) & -\omega B^{k}(t) K^{k}(n T) & \omega A^{k}(t) & 0 & A^{k l}(t) \\
0 & 0 & 0 & \omega A^{k}(t) & A^{k l}(t) \\
\omega \Upsilon_{1}^{k}(t) & -\omega B^{k}(t) K^{k}(n T) & \omega A^{k}(t) & 0 & A^{k l}(t) \\
0 & 0 & 0 & \omega A^{k}(t) & A^{k l}(t)
\end{array}\right],
$$

$\hat{H}_{22}^{k}=\operatorname{diag}\left\{-T^{-1} \omega\left(P_{11}^{k}\right)^{-1},-R_{2}^{k},-T^{-2} \omega^{2}\left(P_{21}^{k}\right)^{-1},-R_{3}^{k}\right\}$.

By using the congruence transformation with diag \{ $\left.\left(P_{11}^{k}\right)^{-1},\left(P_{11}^{k}\right)^{-1},\left(P_{21}^{k}\right)^{-1},\left(R_{1}^{k}\right)^{-1},\left(R_{3}^{k}\right)^{-1}, I, I, I, I\right\}$, applying the Schur complement and denoting $\left(P_{11}^{k}\right)^{-1}=Q_{1}^{k},\left(P_{21}^{k}\right)^{-1}=Q_{2}^{k}$, $\left(P_{3}^{k}\right)^{-1}=Q_{3}^{k}, K_{i}^{k}\left(P_{11}^{k}\right)^{-1}=M_{i}^{k}$, inequality (23) can be represented as

$$
\sum_{i=1}^{r} \sum_{j=1}^{r} \mu_{i}^{k}\left(z_{k}(t)\right) \mu_{j}^{k}\left(z_{k}(n T)\right) \mathcal{X}_{i j}^{k l} \prec 0
$$

Also, by using the Schur complement, applying Lemma 3 and denoting $P_{12}^{k} L_{i}^{k}=N_{i}^{k}$, inequality (22) can be converted into LMI (20). Thus, if LMIs (19) and (20) are satisfied, inequality (8) of Proposition 2 is also satisfied, and the whole closed-loop system (4) is asymptotically stable.

Remark 2: In Theorems 1, we assume that the parameters $\alpha_{k}, \beta_{k}, \gamma_{1_{k}}$ and $\gamma_{2_{k}}$ are given in advance. However, when these parameters are unknown, the parameter value must first be determined. In this case, the iterative LMI (ILMI), which is minutely described in [12], has to be used.

\section{An illustrative example}

In this section, an example is given to validate the proposed sampled-data observer-based decentralized fuzzy control method. Suppose the double Chua's circuit system $[30,31]$ connected by a resistor as follows:

$$
\begin{aligned}
& \dot{v}_{k_{1}}(t)=\frac{1}{C_{k_{1}}}\left(\frac{1}{R_{k}}\left(v_{k_{2}}(t)-v_{k_{1}}(t)\right)-f_{k}\left(v_{k_{1}}(t)\right)+\frac{1}{R_{a}}\left(v_{l_{1}}(t)-v_{k_{1}}(t)\right)+u_{k_{1}}(t)\right) \\
& \dot{v}_{k_{2}}(t)=\frac{1}{C_{k_{1}}}\left(\frac{1}{R_{k}}\left(v_{k_{1}}(t)-v_{k_{2}}(t)\right)-i_{k}(t)+\sigma_{k_{1}} u_{k_{2}}(t)\right) \\
& \dot{i}_{k}(t)=-\frac{1}{L_{k}}\left(v_{k_{2}}(t)+\sigma_{k_{2}} u_{k_{3}}(t)\right), y_{k}(t)=y_{k}(n T)=v_{k_{1}}(n T),
\end{aligned}
$$

where $\left\{(k, l) \in \mathcal{I}_{2} \mid k \neq l\right\} ; \quad v_{k_{1}}(t), \quad v_{k_{2}}(t)$ and $i_{k}(t)$ are state variables of the $k$ th Chua's circuit; $R_{k}$ is a resistor with $R_{1}=100 m \Omega$ and $R_{2}=125 \mathrm{~m} \Omega ; C_{k_{1}}$ and $C_{k_{2}}$ are capacitors with $C_{k_{1}}=1 F$ and $C_{k_{2}}=10 F ; L_{k}$ is an inductor with $L_{1}=70 \mathrm{mH}$ and $L_{2}=75 \mathrm{mH} ; \sigma_{k_{1}}$ and $\sigma_{k_{2}}$ are input coefficients with $\sigma_{1_{1}}=10, \sigma_{1_{2}}=10, \sigma_{2_{1}}=0.07$ and $\sigma_{2_{2}}=0.075 ; R_{a}=3 \Omega$ is an interconnected resistor between circuits; and $f_{k}\left(v_{k_{1}}(t)\right)=\left(g_{b} / R_{k}\right) v_{k_{1}}(t)$ $+\left(\left(g_{a}-g_{b}\right) / 2 R_{k}\right)\left(\left|v_{k_{1}}(t)+1\right|-\left|v_{k_{1}}(t)-1\right|\right) \quad$ is a Chua's diode with $g_{a}=-1.27$ and $g_{b}=-0.68$.

By choosing $x_{k}(t)=\left[\begin{array}{lll}v_{k_{1}}(t) & v_{k_{2}}(t) & i_{k}(t)\end{array}\right]^{T}$ and $u_{k}(t)$

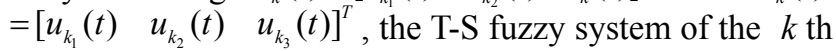
subsystem can be constructed as follows:

$$
\begin{aligned}
& \dot{x}_{k}(t)=\sum_{i=1}^{2} \mu_{i}^{k}\left(x_{k_{1}}(t)\right)\left(A_{i}^{k} x_{k}(t)+B_{i}^{k} u_{k}(t)+\sum_{l \neq k}^{2} A_{i}^{k l} x_{l}(t)\right) \\
& y_{k}(t)=y_{k}(n T)=\sum_{i=1}^{2} \mu_{i}^{k}\left(x_{k_{1}}(n T)\right) C_{i}^{k} x_{k}(n T)
\end{aligned}
$$

where

$$
\begin{aligned}
& A_{1}^{k}=\left[\begin{array}{ccc}
(d-1) \lambda_{k_{1}}-\lambda_{k_{3}} & \lambda_{k_{1}} & 0 \\
1 & -1 & 1 \\
0 & -\lambda_{k_{2}} & 0
\end{array}\right], \\
& A_{2}^{k}=\left[\begin{array}{ccc}
-(d+1) \lambda_{k_{1}}-\lambda_{k_{3}} & \lambda_{k_{1}} & 0 \\
1 & -1 & 1 \\
0 & -\lambda_{k_{2}} & 0
\end{array}\right], \\
& A_{i}^{k l}=\left[\begin{array}{ccc}
\lambda_{k_{3}} & 0 & 0 \\
0 & 0 & 0 \\
0 & 0 & 0
\end{array}\right], B_{i}^{k}=\left[\begin{array}{lll}
1 & 0 & 0 \\
0 & 1 & 0 \\
0 & 0 & 1
\end{array}\right], \\
& C_{i}^{k}=\left[\begin{array}{lll}
1 & 0 & 0
\end{array}\right], \\
& \mu_{1}^{k}\left(x_{k_{1}}(t)\right)=\left\{\begin{array}{l}
1 \\
\frac{1}{2}\left(1-\left(f_{k}\left(x_{k_{1}}(t)\right) / d x_{k_{1}}(t)\right)\right), \\
g_{a}
\end{array}\right. \\
& \mu_{2}^{k}\left(x_{k_{1}}(t)\right)=1-\mu_{1}^{k}\left(x_{k_{1}}(t)\right)
\end{aligned}
$$

with the parameters $\lambda_{1_{1}}=10, \lambda_{1_{2}}=14.2857, \lambda_{2_{1}}=8$, $\lambda_{2_{2}}=13.3333, \quad \lambda_{3_{1}}=\lambda_{3_{2}}=0.3333$ and $d=1.8$ for 
$(i, k, l) \in \mathcal{I}_{2} \times \mathcal{I}_{2} \times \mathcal{I}_{2}$ with $k \neq l$. In this simulation model, the premise variable $x_{k_{1}}(t)$ can be directly obtained by the output variable.

$$
\begin{aligned}
& K_{1}^{1}=\left[\begin{array}{ccc}
-10.9105 & -9.0469 & 0.0099 \\
-2.6431 & -12.9171 & -0.8660 \\
1.5916 & 12.7990 & -18.1612
\end{array}\right] \text {, } \\
& K_{2}^{1}=\left[\begin{array}{ccc}
-10.9105 & -9.0469 & 0.0099 \\
-2.6431 & -12.9171 & -0.8660 \\
1.5916 & 12.7990 & -18.1612
\end{array}\right] \text {, } \\
& K_{1}^{2}=\left[\begin{array}{ccc}
-15.6162 & -7.1073 & 0.0049 \\
-4.5550 & -13.1838 & -0.7869 \\
3.2756 & 11.3915 & -26.1455
\end{array}\right] \text {, } \\
& K_{2}^{2}=\left[\begin{array}{ccc}
-15.6162 & -7.1073 & 0.0049 \\
-4.5550 & -13.1838 & -0.7869 \\
3.2756 & 11.3915 & -26.1455
\end{array}\right] \text {, } \\
& L_{1}^{1}=\left[\begin{array}{c}
67.5308 \\
49.9071 \\
-2.7486
\end{array}\right], \quad L_{2}^{1}=\left[\begin{array}{c}
31.5308 \\
49.9071 \\
-2.7486
\end{array}\right] \text {, }
\end{aligned}
$$

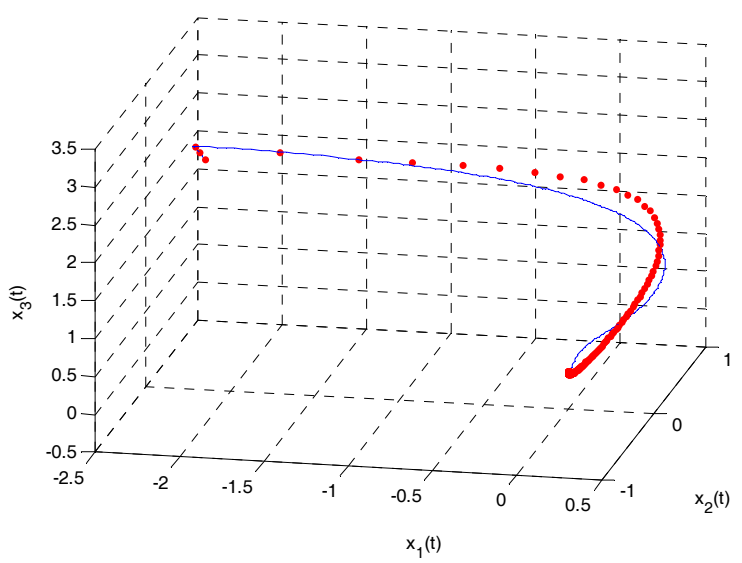

Fig. 1. The time response of large-scale system: subsystem 1 (solid) and subsystem 2 (dash-dotted).

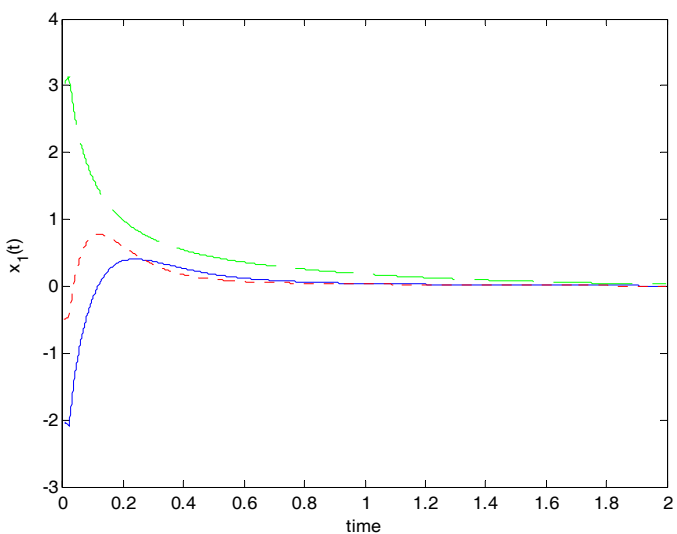

Fig. 2. The time response of subsystem 1 for $T=0.02 \mathrm{~s}$ : $x_{1_{1}}(t)$ (solid), $x_{1_{2}}(t)$ (dotted) and $x_{1_{3}}(t)$ (dashed).

$$
L_{1}^{2}=\left[\begin{array}{l}
62.4354 \\
35.6605 \\
-4.2859
\end{array}\right], \quad L_{2}^{2}=\left[\begin{array}{l}
32.0354 \\
35.6605 \\
-4.2859
\end{array}\right] \text {. }
$$

Based on the above control and observer gains, we obtain the closed-loop large-scale systems and present the

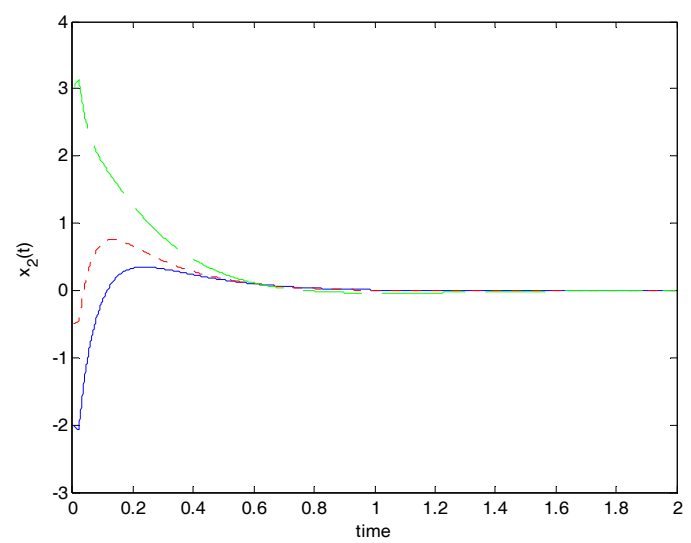

Fig. 3. The time response of subsystem 2 for $T=0.02 \mathrm{~s}$ : $x_{2_{1}}(t)$ (solid), $x_{2_{2}}(t)$ (dotted) and $x_{2_{3}}(t)$ (dashed).

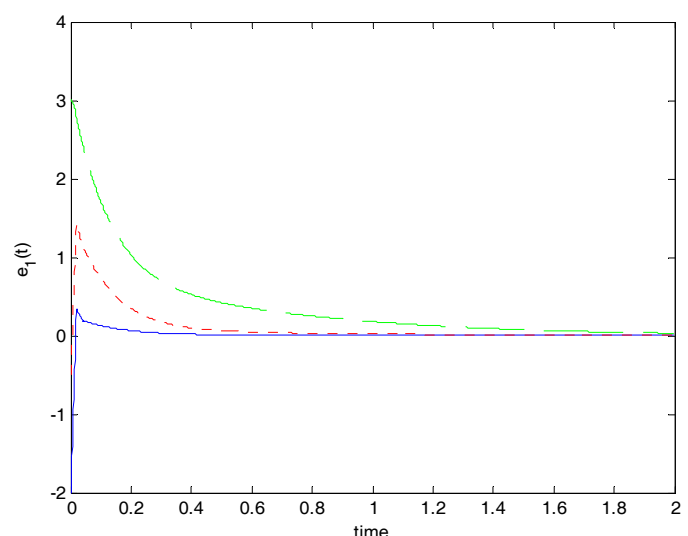

Fig. 4. The estimated error of subsystem 1 for $T=0.02 \mathrm{~s}$ : $e_{1_{1}}(t)$ (solid), $e_{1_{2}}(t)$ (dotted) and $e_{1_{3}}(t)$ (dashed).

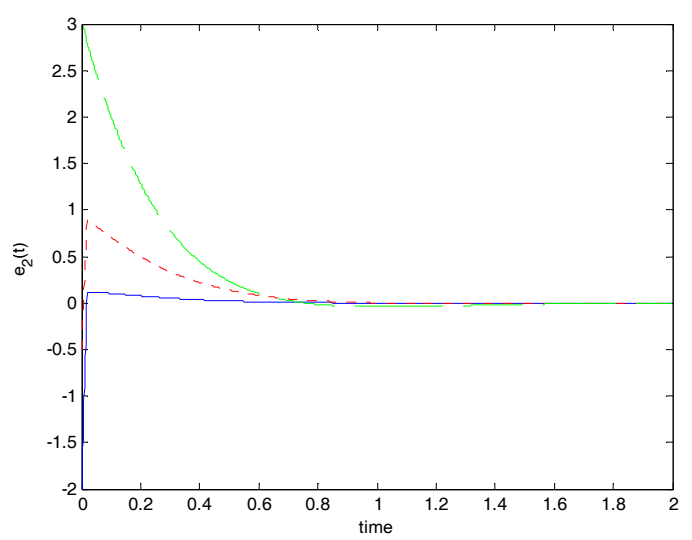

Fig. 5. The estimated error of subsystem 2 for $T=0.02 \mathrm{~s}$ : $e_{2_{1}}(t)$ (solid), $e_{2_{2}}(t)$ (dotted) and $e_{2_{3}}(t)$ (dashed). 
time responses of the state variables and estimated errors for each subsystem. The time responses are shown in Figs. 1, 2, 3, 4 and 5. As shown in the figures, all state variables and errors are converted to 0 , and it means that the obtained gains are suitable to stabilize the double Chua's circuit system. Thus, we know that the proposed sampleddata observer-based decentralized fuzzy control technique is suitable for a nonlinear large-scale system.

\section{Conclusions}

This paper has established a sampled-data observerbased decentralized fuzzy controller for nonlinear largescale systems. Based on the T-S fuzzy system, the closedloop systems have been represented. The sufficient condition has been derived for the stability of the closedloop system by using the exact discretized model and the discrete-time Lyapunov functional and was formulated in the LMI formats. Finally, the numerical example was provided to demonstrate the effectiveness of the proposed techniques.

\section{Acknowledgements}

This work was supported by the National Research Foundation of Korea (NRF) grant funded by the Korea government (MEST) (NRF-2015R1A2A2A05001610)

\section{References}

[1] M. Jamshidi, Large-Scale System: Modeling and Control: Elesevier, 1983.

[2] T. Chen and B. A. Francis, Optimal Sampled-Data Control Systems: Springer, 1995.

[3] L. Bakule, "Decentralized control: an overview," Annual Reviews in Control, vol. 32, pp. 87-98, 2008.

[4] S. S. Stanković, D. M. Stipanović, and D. D. Šiljak, "Decentralized dynamic output feedback for robust stabilization of a class of nonlinear interconnected systems," Automatica, vol. 43, pp. 861-867, 2007.

[5] S. S. Stanković and D. D. Šiljak, "Robust stabilization of nonlinear interconnected systems by decentralized dynamic output feedback," Syst. Control Lett., vol. 58, pp. 271-275, 2009.

[6] S. Tong, C. Liu, and Y. Li, "Fuzzy-adaptive decentralized output-feedback control for large-scale nonlinear systems with dynamical uncertainties," IEEE Trans. Fuzzy Syst., vol. 18, no. 5, pp. 845-861, 2010.

[7] S. Ganapathy and S. Velusami, "Decentralized loadfrequency control of interconnected power systems with SMES units and governor dead band using multi-objective evolutionary algorithm," J. Electr. Eng. \& Technol. vol. 4, no. 4, pp. 443-450, 2009.
[8] D. Nešić and A. R. Teel, "Stabilization of sampleddata nonlinear systems via backstepping on their Euler approximate model," Automatica, vol. 42, pp. 18011808, 2006.

[9] Z. Mao, B. Jiang, and P. Shi, "Fault-tolerant control for a class of nonlinear sampled-data systems via a Euler approximate observer," Automatica, vol. 46, pp. 1852-1859, 2010.

[10] E. Fridman, U. Shaked, and V. Suplin, "Input/output delay approach to robust sampled-data $H_{\infty}$ control," Syst. Control Lett., vol.54, pp.271-282, 2005.

[11] E. Fridman, "A refined input delay approach to sampled-data control," Automatica, vol.46, pp.421427, 2010.

[12] C.-S. Tseng, "A novel approach to $H_{\infty}$ de-centralized fuzzy-observer-based fuzzy control design for non-linear interconnected systems," IEEE Trans. Fuzzy Syst., vol. 16, no. 5, pp. 1337-1350, 2008.

[13] F.-H. Hsiao, J.-D. Hwang, C.-W. Chen, and Z.-R. Tsai, "Robust stabilization of nonlinear multiple timedelay large-scale systems via decentralized fuzzy control," IEEE Trans. Fuzzy Syst., vol. 13, no. 1, pp. 152-163, 2005.

[14] C. Hua and S. X. Ding, "Decentralized networked control system design using T-S fuzzy approach," IEEE Trans. Fuzzy Syst., vol. 20, no. 1, pp. 9-21, 2012.

[15] G. B. Koo, J. B. Park, and Y. H. Joo, "Decentralized fuzzy observer-based output-feedback control for nonlinear large-scale systems: an LMI approach," IEEE Trans. Fuzzy Syst., vol. 22, no. 2, pp. 406-419, 2014.

[16] H. K. Lam and H. F. Leung, "Sampled-data fuzzy controller for time-delay nonlinear systems: fuzzymodel-based LMI approach," IEEE Trans. Syst, Man, Cybern. B, Cybern., vol.37, no.3, pp.617-629, 2007.

[17] H. K. Lam and L. D. Seneviratne, "Chaotic synchronization using sampled-data fuzzy controller based on fuzzy-model-based approach," IEEE Trans. Fuzzy Syst., vol. 55, no. 3, pp. 883-892, 2008.

[18] D. H. Lee, J. B. Park, Y. H. Joo, and S. K. Kim, "Local Hळ Controller Design for Continuous-time TS Fuzzy Systems", Int. J. Control, Autom. Syst., Vol. 13, No. 6, pp. 1499-1507, 2015, 12.

[19] J. Yoneyama, "Robust sampled-data stabilization of uncertain fuzzy systems via input delay approach," Inform. Sciences, vol. 198, pp. 169-176, 2012.

[20] H. J. Lee, J. B. Park, and Y. H. Joo, "Digitalizing a fuzzy observer-based output-feedback control: intelligent digital redesign approach," IEEE Trans. Fuzzy Syst., vol. 13, no. 5, pp. 701-716, 2005.

[21] H.C. Sung, D.W. Kim, J.B. Park, and Y.H. Joo, "Robust digital control of fuzzy systems with parametric uncertainties: LMI-based digital redesign approach," Fuzzy Sets Syst., vol. 161, pp. 919-933, 2010.

[22] G. B. Koo, J. B. Park, and Y. H. Joo, "Intelligent digital redesign for nonlinear interconnected systems using decentralized fuzzy control," J. Electr. Eng. \& 
Technol., vol. 7, no. 3, pp. 420-428, 2012.

[23] D. W. Kim, J. B. Park, and Y. H. Joo, "Theoretical justification of approximate norm minimization method for intelligent digital redesign," Automatica, vol. 44, pp. 851-856, 2008.

[24] H. J. Lee and D. W. Kim, "Intelligent digital revisited: approximate discretization and stability limitation," Fuzzy Sets Syst., vol.159, pp.3221-3231, 2008.

[25] D. W. Kim, H. J. Lee, and M. Tomizuka, "Fuzzy stabilization of nonlinear systems under sampled-data feedback: an exact discrete-time model approach," IEEE Trans. Fuzzy Syst., vol. 18, no. 2, pp. 251260, 2010.

[26] D. W. Kim and H. J. Lee, "Sampled-data observerbased output-feedback fuzzy stabilization of nonlinear systems: exact discrete-time design approach," Fuzzy Sets Syst., vol. 201, pp. 20-39, 2012.

[27] G. B. Koo, J. B. Park, and Y. H. Joo, "Guaranteed cost sampled-data fuzzy control for non-linear systems: a continuous-time Lyapunov approach," IET Control Theory Appl., vol. 13, no. 7, pp. 1745-1752, 2013.

[28] K. Gu, "An integral in the stability problem of timedelay systems," in Proceedings of 39th IEEE Conference on Decision and Control, Sydney, Australia, December 2000.

[29] J. V. D. Oliveira, J. Bernussou, and J. C. Geromel, “A new discrete-time robust stability condition," Syst. Control Lett., vol. 37, pp. 261-265, 1999.

[30] Y. Zheng and G. Chen, "Fuzzy impulsive control of chaotic based on TS fuzzy model," Chaos, Solitons Fractals, vol. 39, pp. 2002-2011, 2009.

[31] W.-S. Yu and T.-S. Wu, "Fuzzy adaptive observerbased control for Chua's circuit with output time delay," IET Control Theory Appl., vol. 5, no. 4, pp. 303-320, 2011.

[32] H. C. Sung, J. B. Park, “Observe-based sampled-data control for uncertain nonlinear systems: intelligent digital redesign approach," Int. J. Control, Autom. Syst., vol. 12, No. 3, pp. 486-496, 2014, 6.

[33] D. H. Lee, Y. H. Joo, and S. K. Kim, " $H_{\infty}$ Digital Redesign for LTI Systems", Int. J. Control, Autom. Syst., Vol. 13, No. 3. pp. 603-610, 2015, 6.

[34] D. H. Lee, Y. H. Joo, and M. H. Tak, "LMI Conditions for Local Stability and Stabilization of Continuous-Time T-S Fuzzy Systems", Int. J. Control, Autom. Syst., Vol. 13, No. 4, pp. 986-994, 2015, 8.

[35] D. H. Lee and Y. H. Joo, "LMI-based Robust Sampled-data Stabilization of Polytopic LTI Systems: A Truncated Power Series Expansion Approach", Int. J. Control, Autom. Syst., vol., 3, no. 2, pp. 284-291, 2015,4 .

[36] D. H. Lee, Y. H. Joo, and S. K. Kim, "FIR-type Robust $\mathrm{H} 2$ and $\mathrm{H}_{\infty}$ Control of Discrete Linear Timeinvariant Polytopic Systems via Memory Statefeedback Control Laws”, Int. J. Control, Autom. Syst., Vol. 13, No. 5, pp. 1047-1056, 2015, 10.

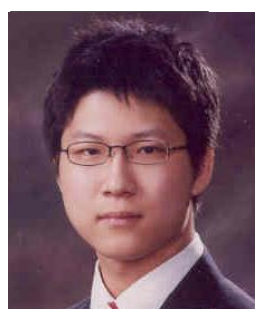

Geun Bum Koo He received the B.S. and Ph.D. degrees in Electrical and Electronic Engineering from Yonsei University Seoul, Korea in 2007 and 2015, respectively. His current research interests include fuzzy systems, decentralized control, and intelligent digital redesign.

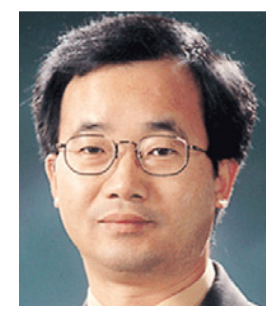

Jin Bae Park He received the B.S. degree in Electrical Engineering from Yonsei University, Seoul, Korea, and the M.S. and Ph.D. degrees in Electrical Engineering from Kansas State University, Manhattan, KS, USA in 1977, 1985, and 1990, respectively. Since 1992, he has been with the Department of Electrical and Electronic Engineering, Yonsei University, Seoul, Korea, where he is currently a professor. His major research interests include robust control and filtering, nonlinear control, intelligent mobile robot, drone, fuzzy logic control, neural networks, adaptive dynamic programming, chaos theory, and genetic algorithms. He served as the Editor-in-Chief for the International Journal of Control, Automation, and Systems (IJCAS) (2006-2010) and the President for the Institute of Control, Robot, and Systems Engineers (ICROS) (2013). He is currently serving as the Senior Vice-President for Yonsei University.

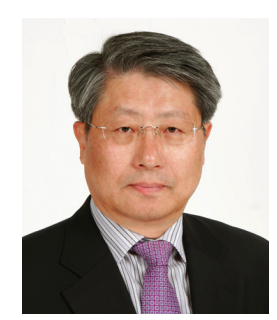

Young Hoon Joo received the B.S., M.S., and Ph.D. degrees in Electrical Engineering from Yonsei University, Seoul, Korea, in 1982, 1984, and 1995, respectively. He worked with Samsung Electronics Company, Seoul, Korea, from 1986 to 1995, as a project manager. He was with the University of Houston, Houston, TX, from 1998 to 1999, as a visiting professor in the Department of Electrical and Computer Engineering. He is currently a professor in the Department of Control and Robotics Engineering, Kunsan National University, Korea. His major interest is mainly in the field of intelligent robot, intelligent control, robot vision, human-robot interaction, wind farm control, and intelligent surveillance systems. He severed as President for Korea Institute of Intelligent Systems (KIIS) (2008-2009) and as the Vice-President for the Korean Institute of Electrical Engineers (KIEE) (2013-2014) and is serving as Editor-inChief for the Intelligent Journal of Control, Automation, and Systems (IJCAS) (2014-present). 